\title{
MONITORING THE COMMUNICATION CHANNEL FROM PUSCHSHINO TO MOSCOW IN THE PROJECT OF SPACE RADIO TELESCOPE "RADIOASTRON"
}

\author{
D.V. Dumsky ${ }^{1,2}$, E.A. Isaev ${ }^{1,2}$, V.A. Samodurov ${ }^{1,2}$, K.A. Isaev ${ }^{3}$ \\ 1 P.N. Lebedev Physical Institute, RAS, \\ 53 Leninskiy Prospekt, Moscow, 119991, Russia,dumsky@prao.ru \\ 2 National research university Higher school of economics, is@itaec.ru \\ Moscow, 101000, Russia \\ ${ }^{3}$ Institute of Mathematical Problems of Biology, RAS, \\ 4, Institutskaja str., Pushchino, Moscow Region, 142290, Russia
}

\begin{abstract}
The need for transmission and storage of large amounts of scientific data in the project space radio telescope "Radioastron" required us to organize a reliable communication channel between the tracking station in Pushchino and treatment centers in Moscow. Network management data requires us to an integrated approach and covers the organization secure access to manage network devices, timely replacement of equipment and software upgrades, backups, as well as documentation of the network infrastructure. The reliability of the channel is highly dependent on continuous monitoring of network and server equipment and communication lines.
\end{abstract}

Keywords: Radioastron; monitoring; telecommunications.

\section{Introduction}

The RadioAstron is an international space VLBI project led by the Astro Space Center of Lebedev Physical Institute in Moscow, Russia. The payload Space Radio Telescope, is based on spacecraft Spektr$\mathrm{R}$, that have been designed by the Lavochkin Associations. The purpose of the project is to use the space telescope for radio astronomical observations using VLBI (Very Long Baseline Interferometry) techniques in tandem with ground-based VLBI networks located in Australia, Chile, China, Europe, India, Japan, Korea, Mexico, Russia, South Africa, Ukraine, and the USA. The scientific objective of the project is to produce an image coordinate measurement and angular movements of different celestial radio sources with extremely high angular resolution (Kardashev et. al., 2012, Kanevskiy et al., 2014).

Ground tracking support of the spacecraft is currently provided using the 22-m antenna (RT-22) located at the radio observatory near Puschino, outside of Moscow (Fig.1). Flow of digital data received by tracking station can reach $128 \mathrm{Mbit} / \mathrm{s}$. For efficient data exchange the direct communication fiber channel between PRAO and processing center ASC FIAN was created. The capacity of channel is $1 \mathrm{Gbit} / \mathrm{sec}$. In addition to tracking station the buffer data-center created as a reference node communication and file storage accomodation. The scientific data channel is used for transmission of telemetry information from the board of the spacecraft too. The channel of communication provided by ISP with guarantee of redundancy via two independent optical lines, and organized using technology MPLS (Multiprotocol Label Switching).

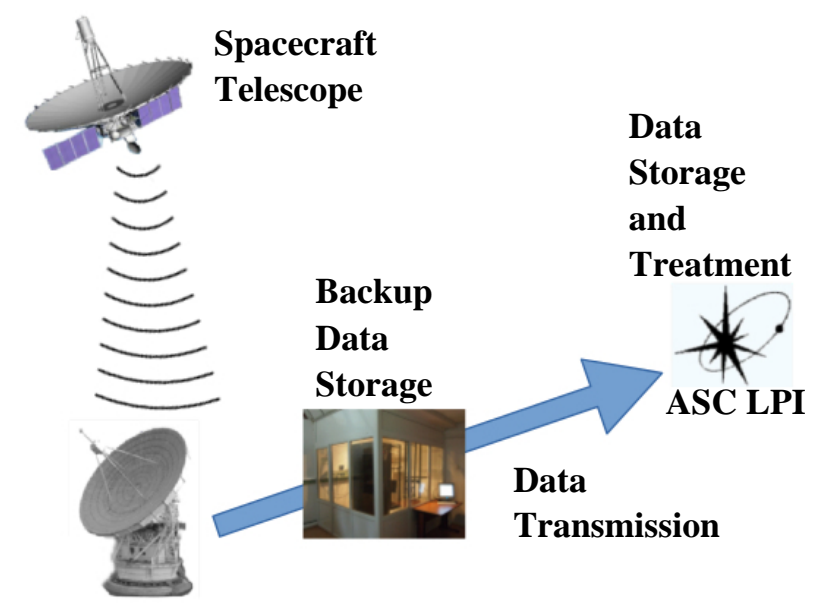

RT22 data acquisition

Figure 1: Radioastron data chanells. 


\section{Chanell monitoring}

Failsafe operation of the communication channel is especially relevant for the space telescope. For example, uninterrupted reception of telemetry data allows specialists to take further decisions on the correction of its orbit and receive important information about the performance of the onboard equipment. The success of the scientific experiments directly related to the reliability of the transmission of scientific data. This requires solutions that can automatically detect and respond to threats and channel performance issues in real time, as well as predict possible issues in the future. Monitoring system gives us the opportunity to have the right information about data transmission at the right time.

Real-time monitoring of hundred of metrics collected from network devices and servers is an important prerequisite for the smooth operation of the scientific data channel and the equipment in its structure. Monitor of availability, syslog messages, power consumption, temperature conditions and other various aspects of equipment performance help us maintained overall performance of the channel at an acceptable level. The main features of monitoring system is alerts when a fault occurs and storage of events history in DB.

We choose Zabbix as monitoring system because it is easy to use, it is Open Source and met most of the requirements we had for a monitoring tool in our environment for channel maintenance (Dumsky et al., 2014). The monitiring system is a powerful network tool that helps us to ensure that critical systems, applications and services are alwaysup and running. It must provides features such as alerting, event handling, reporting and performance statistics. With items collecting data and triggers designed to "fire" upon problem situations, we have alerting mechanism in place that is notify us about important events by email even when we are not directly looking at Zabbix frontend panel. We can see in which of the sites of the channel detects a problem with the help of interactive map monitoring in real-time. The current values of monitored parameters, as well as their changes can be viewed as separate graphs with scale changes by the hour, day, week and month (Fig.2).

\section{Conclusion}

Every day about 50 to 300 GB of unique information about the structure and evolution of various astrophysical objects (blazars, pulsars, cosmic masers and black holes) is transmitted through the channel. The same amount of data backed up in the PRAO ASC LPI buffer data storage. The average data rate of the channel transmission is typically 300-400 Gbit/s.

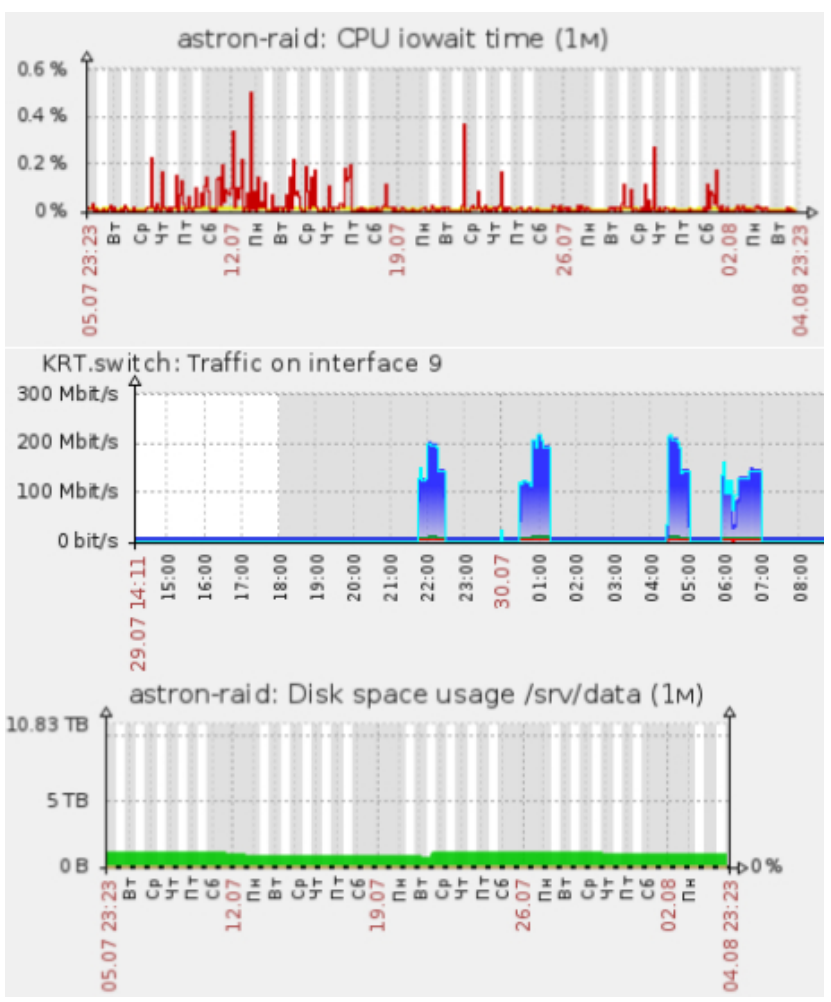

Figure 2: Graphs of important channel parameters.

The monitoring system over the past two years, allows us quickly resolved emergency situations caused by the power failure and failures of the network equipment, and cooling system of the buffer data center, as well as identify the causes of failures of channel associated with the failure of the individual switching devices and optical modules. We were promptly detected and eliminated two cases of the optical lines damage, allowing to minimize the idle time of the channel work. Monitoring of disks in buffer storage allows us for timely replacement of failed drives, that would prevent the destruction of RAID-array and prevent permanent loss of scientific data.

\section{References}

Kardashev N.S., Kovalev Y.Y., Kellermann K.I.: 2012, The URSI Radio Science Bulletin, 343, 22.

Kanevskiy B.Z. et al.: 2014, Bulletin of S.A.Lavoshkin Federal Research and Production Association, 3, 47.

Dumsky D., Isaev E., Samodurov V.A. et al.: 2014, Odessa Astron. Publ., 27. 71. 